\title{
Moving knowledge into practice: Evaluating cross- cultural applicability of the Promoting Action on Research Implementation in Health Service framework
}

\author{
Author: Liz Avital
}

\begin{abstract}
Aims
To explore the appropriateness and utility of the Promoting Action on Research Implementation in Health Service (PARIHS) framework in the cultural translation and adaptation of an evidence-based clinical practice guideline into clinical practice in the healthcare system in Malta. To identify the challenges and barriers to successful cultural translation and implementation to inform future cross-cultural knowledge translation programmes.
\end{abstract}

\section{Methods}

This was an embedded single case, case study. Data collection was facilitated using focus groups with multidisciplinary healthcare professionals, semi-structured interviews, non-participant observation of two guideline development groups. Data were analysed both inductively and deductively using Framework Analysis. Guideline development was based on NICE methodology.

\section{Results}

Findings indicate that the components of the PARIHS framework of evidence, context and facilitation are useful to guide the cultural translation and adaptation of an evidence-based clinical practice guideline. Challenges and barriers to successful translation and potential implementation were identified; the influence of politics, culture and context, resources; human and financial and stakeholder involvement.

\section{Conclusion}

The PARIHS framework can be used to guide the cultural translation and adaptation of an evidence-based clinical practice guideline in a cross-cultural setting. For the PARIHS framework to be fully applicable it is suggested that a number of adaptations are made, primarily:

$>$ the definition of context needs to be expanded to include the wider social / political / organisational context

$>$ culture needs to be a standalone sub element of context to understand the multifaceted role it has
> the role of the patient and the influence they can have needs to be included

> politics should be included as a sub element of context

$>$ the influential role of resources, both human and financial needs to be better recognised.

\section{Conflict of interest statement}

There are no conflicts of interest. 